\title{
On Semiparallel and Weyl-semiparallel Hypersurfaces of Kaehler Manifolds
}

CIHAN ÖZGÜR*

Department of Mathematics, Balıkesir University 10145, Ballkesir, TURKEY

e-mail : cozgur@balikesir.edu.tr

Cengizhan MURATHAN And KAdRi ARSLAN

Department of Mathematics, Uludă̆ University 16059, Bursa, TURKEY

e-mail : cengiz@uludag.edu.tr and arslan@uludag.edu.tr

ABSTRACT. We study on semiparallel and Weyl semiparallel Sasakian hypersurfaces of Kaehler manifolds. We prove that a $(2 n+1)$-dimensional Sasakian hypersurface $M$ of a $(2 n+2)$-dimensional Kaehler manifold $\widetilde{M}^{2 n+2}$ is semiparallel if and only if it is totally umbilical with unit mean curvature, if $\operatorname{dim} M=3$ and $\widetilde{M}^{4}$ is a Calabi-Yau manifold, then $\widetilde{M}$ is flat at each point of $M$. We also prove that such a hypersurface $M$ is Weyl-semiparallel if and only if it is either an $\eta$-Einstein manifold or semiparallel. We also investigate the extended classes of semiparallel and Weyl semiparallel Sasakian hypersurfaces of Kaehler manifolds.

\section{Introduction}

Given an isometric immersion $f: M \longrightarrow \tilde{M}$, let $h$ be the second fundamental form and $\bar{\nabla}$ the van der Waerden-Bortolotti connection of $M$. J. Deprez defined the immersion to be semiparallel if

$$
\bar{R}(X, Y) \cdot h=\left(\bar{\nabla}_{X} \bar{\nabla}_{Y}-\bar{\nabla}_{Y} \bar{\nabla}_{X}-\bar{\nabla}_{[X, Y]}\right) h=0
$$

holds for all vector fields $X, Y$ tangent to $M$, where $\bar{\nabla}$ denotes van der WaerdenBortolotti connection of $M$ and $\bar{R}$ the curvature tensor of $\bar{\nabla}$. In [5] and [6], J. Deprez studied semiparallel immersions in real space forms. In [10], ய̈. Lumiste showed that a semiparallel submanifold is the second order envelope of the family of parallel submanifolds. In [9], in the case of hypersurfaces in the sphere and the hyperbolic space, F. Dillen showed that semiparallel hypersurfaces are flat surfaces, hypersurfaces with parallel Weingarten endomorphism or rotation hypersurfaces of certain helices. In [8], R. Deszcz, L. Verstraelen and Ş. Yaprak obtained some results on hypersurfaces in 4-dimensional space form $N^{4}(c)$ satisfying the curvature

\footnotetext{
* Corresponding author.
}

Received 11 February 2008; accepted 17 April 2008.

2000 Mathematics Subject Classification: 53C15, 53C55, 53C25, 53C42.

Key words and phrases: Sasakian manifold, Kaehler manifold, Calabi-Yau manifold, semiparallel submanifold, Weyl-semiparallel submanifold, pseudoparallel submanifold. 
condition

$$
\bar{R} \cdot h=L_{h} Q(g, h),
$$

where $L_{h}$ is some function on the hypersurface. A. C. Asperti, G. A. Lobos and F. Mercuri defined these type submanifolds as pseudoparallel (see [1] and [2]). In [2], it was shown that a pseudoparallel hypersurface of a space form is either quasiumbilical or a cyclic of Dupin. In [11], the first author studied normally flat submanifolds satisfying the condition

$$
C \cdot h=0,
$$

where $C$ denotes the Weyl conformal curvature tensor. This kind of submanifolds are called Weyl-semiparallel. As a generalization of Weyl semiparallelity condition, in [11], it was also considered the condition

$$
C \cdot h=L_{h} Q(g, h),
$$

where $L_{h}$ is some function on the submanifold.

In this paper, we study semiparallel, pseudoparallel and Weyl semiparallel Sasakian hypersurfaces of Kaehler manifolds. We also consider Sasakian hypersurfaces satisfying the condition (4).

The paper is organized as follows: In section 2, we give a brief account of Sasakian manifolds. In section 3, Sasakian hypersurfaces of Kaehler manifolds are presented. In section 4, we classify semiparallel, pseudoparallel and Weyl semiparallel Sasakian hypersurfaces of Kaehler manifolds as main results. We also study Sasakian hypersurfaces of Kaehler manifolds satisfying the condition (4).

\section{Sasakian manifolds}

An $(2 n+1)$-dimensional differentiable manifold $M$ is called an almost contact manifold if there is an almost contact structure $(\varphi, \xi, \eta)$ consisting of a tensor field $\varphi$ of type $(1,1)$, a vector field $\xi$, and a 1-form $\eta$ satisfying

$$
\varphi^{2}=-I+\eta \otimes \xi, \quad \text { and (one of) } \quad \eta(\xi)=1, \quad \varphi \xi=0, \quad \eta \circ \varphi=0 .
$$

If the induced almost complex structure $J$ on the product manifold $M^{2 n+1} \times \mathbb{R}$ defined by

$$
J\left(X, f \frac{d}{d t}\right)=\left(\varphi X-f \xi, \eta(X) \frac{d}{d t}\right)
$$

is integrable then the structure $(\varphi, \xi, \eta)$ is said to be normal, where $X$ is tangent to $M, t$ is the coordinate of $\mathbb{R}$ and $f$ is a smooth function on $M^{2 n+1} \times \mathbb{R} . M$ becomes an almost contact metric manifold with an almost contact metric structure $(\varphi, \xi, \eta, g)$, if

$$
g(\varphi X, \varphi Y)=g(X, Y)-\eta(X) \eta(Y)
$$


or equivalently

$$
g(X, \varphi Y)=-g(\varphi X, Y) \text { and } g(X, \xi)=\eta(X)
$$

for all $X, Y \in T M$, where $g$ is a Riemannian metric tensor of $M$.

An almost contact metric structure is called a contact metric structure if

$$
g(X, \varphi Y)=d \eta(X, Y)
$$

holds on $M$ for $X, Y \in T M$. A normal contact metric manifold is a Sasakian manifold. However an almost contact metric manifold is Sasakian if and only if

$$
\nabla_{X} \varphi=\eta(Y) X-\eta(X) Y, \quad X \in T M
$$

where $\nabla$ is Levi-Civita connection. Also a contact metric manifold $M$ is Sasakian if and only if the curvature tensor $R$ satisfies

$$
R(X, Y) \xi=\eta(Y) X-\eta(X) Y
$$

(see [3]). From (6), by a contraction one can get easily

$$
\mathcal{S} \xi=2 n \xi
$$

and

$$
S(X, \xi)=2 n \eta(X)
$$

where $S$ and $\mathcal{S}$ denotes the Ricci tensor and Ricci operator of $M$, respectively. The Ricci operator $\mathcal{S}$ is defined by

$$
S(X, Y)=g(\mathcal{S} X, Y)
$$

\section{Kaehlerian submanifolds}

Let $\widetilde{M}$ denote a $(2 n+2)$-dimensional Kaehler manifold, i.e., a smooth manifold with a $(1,1)$-tensor field $J$ and a Riemannian metric $g$ such that $J^{2}=-I$, $g(J X, J Y)=g(X, Y), \nabla J=0$ for arbitrary vector fields $X, Y$ on $\widetilde{M}$, where $I$ is identity tensor field and $\nabla$ the Riemannian connection of $g$. Let $M$ be an $(2 n+1)$ dimensional orientable hypersurface isometrically embedded into a Kaehler manifold $\widetilde{M}$. The metrics for both $M$ and $\widetilde{M}$ will be denoted by $g$. The Gauss and Weingarten formulas are given by

$$
\widetilde{\nabla}_{X} Y=\nabla_{X} Y+h(X, Y)
$$

and

$$
\widetilde{\nabla}_{X} N=-A_{N} X+D_{X} \xi
$$


respectively, where $N$ is a normal vector field and $X, Y$ are tangent vector fields on $M$. $h$ is called the second fundamental form of $M$. If $h=0$ then $M$ is said to be totally geodesic. The mean curvature vector $\alpha$ of $M$ is defined to be

$$
\alpha=\frac{1}{2 n+1} \operatorname{tr}(h)
$$

A submanifold $M$ is said to be minimal if $\alpha=0$ identically [4]. Complex manifolds with a Ricci-flat Kaehler metric are called Calabi-Yau manifolds.

From now on we will assume that $M$ is a hypersurface of a Kaehler manifold $\widetilde{M}$. Let $N$ be the unit normal vector field to $M$, then $J N$ is tangent to $M$. We set

$$
\begin{gathered}
J N=\xi, \\
J X=\varphi X-\eta(X) N,
\end{gathered}
$$

where $\varphi$ and $\eta$ denote a $(1,1)$-tensor field and a 1-form respectively, and $X$ an arbitrary tangential vector field on $M$. Since $M$ is a hypersurface of a Kaehler manifold $\widetilde{M}$, the Gauss and Weingarten formulas can be written as

$$
\begin{gathered}
\widetilde{\nabla}_{X} Y=\nabla_{X} Y+H(X, Y) N, \\
\widetilde{\nabla}_{X} N=-A X,
\end{gathered}
$$

where $H$ denotes the second fundamental tensor and $A$ is the shape operator. $H$ and $A$ are related by

$$
H(X, Y)=g(A X, Y),
$$

(see [4]). Differentiating (9) along $M$, using (11) and (12) we get

$$
\nabla_{X} \xi=-\varphi A X .
$$

From (9) and (10), $(\eta, \xi, \varphi, g)$ defines an almost contact metric structure on $M$. Differentiating (10) along $M$, using (11) and (12), and comparing tangential parts we get

$$
\left(\nabla_{X} \varphi\right) Y=H(X, Y) \xi-\eta(Y) A X
$$

(see [12]).

We suppose that the almost contact metric structure induced on $M$ is a contact metric structure. In this study, we consider this contact metric structure as a Sasakian structure. Then by Lemma 6.2 in [3], we have

$$
\nabla_{X} \xi=-\varphi X .
$$

So comparing (15) with (13) we have

$$
A X=X+\eta(A X-X) \xi .
$$


From (16), by a contraction, we have $\operatorname{tr}(A)-2 n=\eta(A \xi)$. Substituting $\xi$ for $X$ in $(16)$ we get

$$
A \xi=(\operatorname{tr}(A)-2 n) \xi
$$

From (16) and (17) we obtain

$$
A X=X+(\operatorname{tr}(A)-2 n-1) \eta(X) \xi,
$$

for the general case see [12].

\section{Main results}

Let $M$ be a $(2 n+1)$-dimensional orientable hypersurface isometrically embedded into a Kaehler manifold $\widetilde{M}$. It is easy to see that for a hypersurface, the conditions $\bar{R} \cdot h=0, \bar{R} \cdot h=L_{h} Q(g, h), C \cdot h=0$ and $C \cdot h=L_{h} Q(g, h)$ can be replaced by $R \cdot H=0, R \cdot H=L_{H} Q(g, H), C \cdot H=0$ and $C \cdot H=L_{H} Q(g, H)$, respectively.

The Weyl conformal curvature tensor [13] of a $(2 n+1)$-dimensional Riemannian manifold is defined by

$$
\begin{aligned}
C(X, Y) Z= & R(X, Y) Z-\frac{1}{2 n-1}\{S(Y, Z) X \\
& -S(X, Z) Y+g(Y, Z) \mathcal{S} X-g(X, Z) \mathcal{S} Y\} \\
& +\frac{r}{2 n(2 n-1)}[g(Y, Z) X-g(X, Z) Y] .
\end{aligned}
$$

The tensors $R \cdot H, C \cdot H$ and $Q(g, H)$ are defined by

$$
\begin{aligned}
& (R(X, Y) \cdot H)(U, V)=-H(R(X, Y) U, V)-H(U, R(X, Y) V), \\
& (C(X, Y) \cdot H)(U, V)=-H(C(X, Y) U, V)-H(U, C(X, Y) V)
\end{aligned}
$$

and

$$
Q(g, H)(U, V ; X, Y)=-H((X \wedge Y) U, V)-H(U,(X \wedge Y) V),
$$

respectively, for vector fields $X, Y, U, V$ tangent to $M$, where $X \wedge Y$ is an endomorphisms defined by

$$
(X \wedge Y) Z=g(Y, Z) X-g(X, Z) Y
$$

and $Z$ is a vector field tangent to $M$ (see [7]).

Firstly, we have the following Theorem:

Theorem 4.1. Let $M$ be a Sasakian hypersurface of a Kaehler manifold $\widetilde{M}^{2 n+2}$. Then $M$ is semiparallel if and only if it is totally umbilical with unit mean curvature. 
Moreover, if $\operatorname{dim} M=3$ and $\widetilde{M}^{4}$ is a Calabi-Yau manifold, then $\widetilde{M}$ is flat at each point of $M$.

Proof. By the use of (20), we can write

$$
(R(X, Y) \cdot H)(U, V)=-g(R(X, Y) U, A V)-g(A U, R(X, Y) V) .
$$

So in view of (18) we get

$$
(R(X, Y) \cdot H)(U, V)=-\lambda[\eta(V) g(R(X, Y) U, \xi)+\eta(U) g(R(X, Y) V, \xi)],
$$

where $\lambda=\operatorname{tr}(A)-2 n-1$. Now suppose that $M$ is semiparallel. Then $R \cdot H=0$ and this implies

$$
\lambda[\eta(V) g(R(X, Y) U, \xi)+\eta(U) g(R(X, Y) V, \xi)]=0 .
$$

Taking $V=\xi$ in (24) we have

$$
\lambda g(R(X, Y) U, \xi)=0 .
$$

Contracting (25) with respect to $X$ and $U$ we get

$$
\lambda S(Y, \xi)=0 .
$$

Taking $Y=\xi$ in (26) and using (7) we find

$$
2 n \lambda=0,
$$

which implies $\lambda=0$. Then from (18) we obtain $A=I$ and so $M$ is totally umbilical with unit mean curvature. Assume that $\operatorname{dim} M=3$ and $\widetilde{M}^{4}$ is a Calabi-Yau manifold. So by the proof of Theorem 2 in [12], we obtain $M$ is of constant curvature 1 and $\widetilde{M}$ has the curvature tensor $\widetilde{R}=0$ at each point of $M$. The converse statement is trivial. Hence we get the result as required.

So we give an extension of this theorem as follows:

Theorem 4.2. Let $M$ be a Sasakian hypersurface of a Kaehler manifold $\widetilde{M}^{2 n+2}$. Then $M$ is pseudoparallel such that $L_{H} \neq 1$ if and only if it is totally umbilical with unit mean curvature. Moreover, if $\operatorname{dim} M=3$ and $\widetilde{M}^{4}$ is a Calabi-Yau manifold, then $\widetilde{M}$ is flat at each point of $M$.

Proof. From (22) and (23) we have

$$
\begin{aligned}
Q(g, H)(U, V ; X, Y)= & -g(Y, U) H(X, V)+g(X, U) H(Y, V) \\
& -g(Y, V) H(X, U)+g(X, V) H(U, Y) .
\end{aligned}
$$

So in view of (18) we get

$$
\begin{gathered}
Q(g, H)(U, V ; X, Y)=\lambda[-g(Y, U) \eta(X) \eta(V)+g(X, U) \eta(Y) \eta(V) \\
-g(Y, V) \eta(X) \eta(U)+g(X, V) \eta(Y) \eta(U)] .
\end{gathered}
$$


Since the condition $R \cdot H=L_{H} Q(g, H)$ holds on $M$, from (24) and (27) we have

$$
\begin{gathered}
\lambda[\eta(V) g(R(X, Y) U, \xi)+\eta(U) g(R(X, Y) V, \xi)]=\lambda L_{H}[-g(Y, U) \eta(X) \eta(V) \\
+g(X, U) \eta(Y) \eta(V)-g(Y, V) \eta(X) \eta(U)+g(X, V) \eta(Y) \eta(U)] .
\end{gathered}
$$

So taking $V=\xi$ in (28) and using (6) we obtain

$$
\lambda\left[L_{H}-1\right][-g(Y, U) \eta(X)+g(X, U) \eta(Y)]=0 .
$$

Since $L_{H} \neq 1$ we find $\lambda=0$. In this case by the proof of Theorem 4.1 we know that $M$ is semiparallel. So the proof follows from the proof of Theorem 4.1.

Conversely, a semiparallel hypersurface is trivially pseudoparallel. This completes the proof of the theorem.

Theorem 4.3. Let $M$ be a Sasakian hypersurface of a Kaehler manifold $\widetilde{M}^{2 n+2}$, $n>1$. Then $M$ is Weyl semiparallel if and only if $M$ is an $\eta$-Einstein manifold or it is totally umbilical with unit mean curvature. Moreover, if $\operatorname{dim} M=3$ and $\widetilde{M}^{4}$ is a Calabi-Yau manifold, then $\widetilde{M}$ is flat at each point of $M$.

Proof. By the use of (21) we can write

$$
(C(X, Y) \cdot H)(U, V)=-g(C(X, Y) U, A V)-g(A U, C(X, Y) V) .
$$

So in view of (18) we get

$$
(C(X, Y) \cdot H)(U, V)=-\lambda[\eta(V) g(C(X, Y) U, \xi)+\eta(U) g(C(X, Y) V, \xi)],
$$

where $\lambda=\operatorname{tr}(A)-2 n-1$. Since $M$ is semiparallel $C \cdot H=0$, which implies

$$
\lambda[\eta(V) g(C(X, Y) U, \xi)+\eta(U) g(C(X, Y) V, \xi)]=0 .
$$

Taking $V=\xi$ in (30) we have

$$
\lambda g(C(X, Y) U, \xi)=0
$$

which gives us either $\lambda=0$ or $g(C(X, Y) U, \xi)$. Assume that $\lambda=0$. So by the proof of previous theorem, we obtain $A=I$ and then $M$ is totally umbilical with unit mean curvature, if $\operatorname{dim} M=3$ and $\widetilde{M}^{4}$ is a Calabi-Yau manifold, then $\widetilde{M}$ is flat at each point of $M$. Now assume that $g(C(X, Y) U, \xi)=0$. Then by the use of (19) and (6) we have

$$
\begin{aligned}
0= & \left(1+\frac{r}{2 n(2 n-1)}\right)(g(Y, U) \eta(X)-g(X, U) \eta(Y)) \\
& -\frac{1}{2 n-1}[S(Y, U) \eta(X)-S(X, U) \eta(Y) \\
& +2 n g(Y, U) \eta(X)-2 n g(X, U) \eta(Y)] .
\end{aligned}
$$


Taking $X=\xi$ in (31) and using (7) we obtain

$$
S(Y, U)=\left(\frac{r}{2 n}-1\right) g(Y, U)+\left(2 n+1-\frac{r}{2 n}\right) \eta(Y) \eta(U),
$$

which gives us $M$ is an $\eta$-Einstein manifold.

Conversely, if $M$ is totally umbilical with unit mean curvature then it can be easily seen that it is Weyl-semiparallel. Now assume that $M$ is an $\eta$-Einstein manifold. Then from (32) and (19) we have

$$
g(C(X, Y) U, \xi)=0 .
$$

So putting (33) into (29) we get $(C(X, Y) \cdot H)(U, V)=0$. This completes the proof of the theorem.

Theorem 4.4. There is no Sasakian hypersurface $M$ of a Kaehler manifold $\widetilde{M}^{2 n+2}$, $n>1$, satisfying the condition $C \cdot H=L_{H} Q(g, H)$ unless $L_{H}=0$.

Proof. Since the condition $C \cdot H=L_{H} Q(g, H)$ holds on $M$ from (27) and (29) we have

$$
\begin{gathered}
-\lambda[\eta(V) g(C(X, Y) U, \xi)+\eta(U) g(C(X, Y) V, \xi)]=L_{H} \lambda[-g(Y, U) \eta(X) \eta(V) \\
+g(X, U) \eta(Y) \eta(V)-g(Y, V) \eta(X) \eta(U)+g(X, V) \eta(Y) \eta(U)] .
\end{gathered}
$$

Then either $\lambda=0$, in which case by the proof of the previous theorem it is easy to see that $C \cdot H=0$, or

$$
\begin{gathered}
-[\eta(V) g(C(X, Y) U, \xi)+\eta(U) g(C(X, Y) V, \xi)]=L_{H}[-g(Y, U) \eta(X) \eta(V) \\
+g(X, U) \eta(Y) \eta(V)-g(Y, V) \eta(X) \eta(U)+g(X, V) \eta(Y) \eta(U)] .
\end{gathered}
$$

Now taking $X=V=\xi$ in (34) we have

$$
\begin{aligned}
S(Y, U)= & \left(\frac{r}{2 n}-1-L_{H}(2 n-1)\right) g(Y, U) \\
& +\left(2 n+1-\frac{r}{2 n}+L_{H}(2 n-1)\right) \eta(Y) \eta(U) .
\end{aligned}
$$

Hence $M$ is an $\eta$-Einstein manifold. From (35), by a contraction, we find

$$
L_{H}(2 n)(2 n-1)=0,
$$

which gives us $L_{H}=0$. This proves the theorem.

Acknowledgment. The authors are thankful to the referee for valuable suggestions towards the improvement of this work. 


\section{References}

[1] A. C. Asperti, G. A. Lobos and F. Mercuri, Pseudo-parallel immersions in space forms, Math. Contemp., 17(1999), 59-70.

[2] A. C. Asperti, G. A. Lobos and F. Mercuri, Pseudo-parallel immersions of a space forms, Adv. Geom., 2(2002) 17(1999), 57-71.

[3] D. E. Blair, Riemannian geometry of contact and symplectic manifolds, Progress in Mathematics, 203. Birkhäuser Boston, Inc., Boston, MA, 2002.

[4] B. Y. Chen, Geometry of submanifolds and its applications, Science University of Tokyo, Tokyo, 1981.

[5] J. Deprez, Semiparallel Surfaces in Euclidean space, J. Geom., 25(1985), 192-200.

[6] J. Deprez, Semiparallel Hypersurfaces, Rend. Sem. Mat. Univers. Politecn. Torino, 44(1986), 303-316.

[7] R. Deszcz, On pseudosymmetric spaces, Bull. Soc. Math. Belg. Sér. A, 44(1)(1992), $1-34$.

[8] R. Deszcz, L. Verstraelen and Ş.Yaprak, Pseudosymmetric hypersurfaces in 4dimensional space of constant curvature, Bull. Ins. Math. Acad. Sinica, 22(1994), $167-179$.

[9] F. Dillen, Semi-parallel hypersurfaces of a real space form, Israel J. Math., 75(1991), 193-202.

[10] Ü. Lumiste, Semi-symmetric submanifolds as the second order envelope of symmetric submanifolds, Proc. Estonian Acad. Sci. Phys. Math., 39(1990), 1-8.

[11] C. Özgür, Submanifolds satisfying some curvature conditions imposed on the Weyl tensor, Bull. Austral. Math. Soc., 67(1)(2003), 95-101.

[12] R. Sharma, Contact hypersurfaces of Kaehler manifolds, J. Geom., 78(1-2)(2003), 156-167.

[13] K. Yano and M. Kon, Structures on manifolds, Series in Pure Mathematics, 3. World Scientific Publishing Co., Singapore, 1984. 